Background Due to the nature and extent of disease, hospice patients are extremely susceptible to infection (Raquel 2005). The decision to commence antimicrobial treatment is often complex (Pereira, Watanabe and Wolfe 1998, Nagy-Agren and Haley,2002). This audit reviews the appropriateness of antimicrobial choice and course length to encourage antimicrobial policy adherence and stewardship

Method A retrospective audit of Antimicrobial prescriptions for patients with life-limiting illness requiring inpatient hospice care at The Royal Trinity Hospice (RTH). Patients admitted over a 3 month period in 2015 were audited. The primary outcome was comparison of antimicrobial choice, dose, course and route against the local DGH Hospital Antibiotic Policy and correlation with trends from an initial audit conducted in 2014.

Results One patient was excluded due to missing documentation; this did not correlate with remarkable antibiotic use. Analysis was limited to 64 prescriptions to include 30 patients. This compared to 58 prescriptions over the complete 3 months in the previous round indicating a significantly increased antimicrobial prescribing rate. Most prevalent infections were UTI (34\%), LRTI (15.6\%) and cellulitis (4.6\%). Our study demonstrated a significant amount of antifungal prescribing (32.8\%), second in prevalence only to the most predominantly treated bacterial infection. There was minimal documentation making analysis of percentage adherence to policy, microbiology consultation and sensitivity requests difficult to interpret with confidence. Trends reflected suboptimal adherence to protocol, with sensitivities requested in $10.9 \%$, all of which were UTI.

Conclusion Most hospices use policies from their local trusts, based on local sensitivities and this is therefore an important and relevant tool. Antimicrobials are frequently prescribed off protocol without clear documentation for the rationale, sensitivity and without microbiological input. There is a tendency towards prescribing augmentin off protocol. Antifungal prescribing policy is poorly represented considering its contribution to microbial burden and quality of life in terminal care.

\section{P-49 'IS HOME WHERE I WANT TO DIE?' - PROGNOSTICATION AND PREFERRED PLACE OF CARE AT THE END OF LIFE IN OLDER HOSPITAL INPATIENTS}

${ }^{1}$ Jacquelyn Stephenson, ${ }^{1}$ Jane Masoli, ${ }^{1,2}$ Maria Leitch, ${ }^{1,2}$ Rebecca Baines . 'Royal Devon and Exeter Hospital, Exeter, UK; ${ }^{2}$ Exeter Hospiscare, Exeter, UK

10.1136/bmjspcare-2017-00133.49

Background Guidelines suggest that the preferred place of care (PPC) for patients at the end of life is in their own home. Existing literature is largely from younger cancer patients. Increasing numbers are living into very old age who may have different care needs and challenges. Studies on PPC and prognostication outside the 'acute' dying phase in older people are limited. We aim to investigate the casemix (cancer vs non-cancer), the PPC and accuracy of prognostication of referrals to the Palliative Discharge Team (PDT).

Methods Analysis of observational database data collected as part of routine clinical practice of the PDT - referred inpatients thought to be in the last 3 months of life. The data were analysed using Stata 14.

Results $n=987$. Mean age at referral 78 years. $60.2 \%$ had palliative cancer diagnoses. The odds ratio of cancer diagnosis decreased with increasing age (OR 0.957 , 95\% CI 0.944 $0.971 ; \mathrm{p} \leq 0.001)$.

Home was the PPC in $34 \%$ patients. Logistic regression analysis found an increased likelihood of change in PPC with age (OR 1.03, 95\% CI 1.02-1.04; $\mathrm{p} \leq 0$.

Mean time from hospital discharge to death was 47 days. $90 \%$ of deaths occurred $<109$ days. No statistically significant difference in time from discharge to death with age or cancer vs non-cancer diagnosis $(\mathrm{p}=0.1684)$.

Discussion Home was not the PPC for the majority of patients and the association of changing PPC with older age and non-cancer diagnosis suggests this group may have different wishes from previous study participants. This is likely to be multifactorial, with different barriers to dying at home in an older population. Cancer dominance of referrals was less prominent in the oldest old. Prognostication was not significantly affected by cancer status and the accuracy suggests underuse of the service. Additional research is required into PPC in older, multi-morbid populations and what factors affect it.

\section{P-50 HYPERCALCAEMIA OF MALIGNANCY: AN ANALYSIS OF THE MEDICAL MANAGEMENT OF PALLIATIVE CANCER PATIENTS IN COMMUNITY, HOSPICE AND HOSPITAL SETTINGS}

${ }^{1}$ Claire McKenzie, ${ }^{2}$ Rachel McDonald, ${ }^{3}$ Amara Nwosu, ${ }^{1}$ Alison White, ${ }^{4}$ Jenny Smith, ${ }^{5}$ Angela Fell, ${ }^{6}$ Marie Keenan, ${ }^{7}$ Melanie Brooks. ${ }^{1}$ St. Rocco's Hospice, Warrington, UK; ${ }^{2}$ Royal Liverpool and Broadgreen University Hospitals NHS Trust, Liverpool, UK; ${ }^{3}$ Marie Curie Palliative Care Institute Liverpool, University of Liverpool, Liverpool, UK; ${ }^{4}$ Countess of Chester Hospital NHS Foundation Trust, Chester, UK; ${ }^{5}$ Lay representative, Warrington, UK; ${ }^{6}$ Warrington and Halton Hospitals NHS Foundation Trust, Warrington, UK; ${ }^{7}$ Bridgewater Community Healthcare Foundation NHS Trust, Warrington, UK

\subsection{6/bmjspcare-2017-00133.50}

Background Hypercalcemia of malignancy (HCM) is a common and significant cause of morbidity and mortality. Treatment includes clinically assisted hydration and bisphosphonates. Denosumab has been used in some centres. Clinical management of hypercalcaemia varies across settings and many recommendations are based on expert opinion.

Aim Within a Regional Palliative Care Clinical Network in the North West of England, we aimed to:

- Evaluate the management of HCM in community, hospice and hospital settings

- Develop new standards and guidelines

Method

- Systematic literature review.

- Six-month retrospective case note analysis of the management of HCM in community, hospice and hospital patients.

- Multi-professional questionnaire survey of palliative care professionals.

Results A systematic literature identified 32 articles to inform development of the regional standards and guidelines. Data for 79 patients was recorded from hospital $(n=53,67 \%)$, hospice $(n=25,32 \%)$ and community $(n=1,1 \%)$ settings. Patients reported high symptomatic burden: fatigue $(n=41,52 \%)$, weakness $(n=38,48 \%)$, drowsiness $(n=32,41 \%)$ and constipation $(n=26,37 \%)$. Intravenous fluids were administered in $72(91 \%)$ patients; $0.9 \%$ saline was most used $(n=67,85 \%)$ within 24 hours of diagnosis $(n=64,81 \%)$. Bisphosphonates were used 